RNA INTERFERENCE

\section{RNAi therapy for preeclampsia}

Haase, N. et al. J. Clin. Invest. https://doi.org/ 10.1172/JCI99417 (2020)

Preeclampsia, a pregnancy complication characterized by new-onset maternal hypertension and proteinuria, is associated with multiple adverse maternal and fetal outcomes. The pathophysiology of preeclampsia remains unclear, but potential contributing factors have been identified, including alterations in the renin-angiotensin system (RAS).

A new study further confirms the role of RAS in preeclampsia by showing that a reduction of maternal hepatic angiotensinogen (AGT), a RAS component, using RNA interference (RNAi) ameliorated the preeclamptic phenotype, reduced blood pressure and improved intrauterine growth restriction in two rodent models of preeclampsia.

Agt RNAi treatment also had no deleterious effects on the fetus. Further investigation is needed to evaluate the clinical potential of this therapy in patients with preeclampsia.

https://doi.org/10.1038/s41684-020-0566-5

\section{EXPERIMENTAL MODEL}

\section{A mouse model for IPF research}

Duerr, J. et al. Nat. Commun. 11, 2012 (2020)

Idiopathic pulmonary fibrosis (IPF) is a progressive lung disease of unknown etiology and with limited therapeutic options. Over the years, progress in IPF research has been hindered by a lack of animal models that recapitulate the progressive evolution of IPF in patients.

A study now shows that conditional deletion of Nedd4-2-a gene encoding E3 ubiquitin-protein ligase NEDD4-like (NEDD4-2), a protein involved in epithelial homeostasis-in lung epithelial cells of mice at adult ages results in a spontaneous and progressive lung disease that shares important features with IPF, including progressive pulmonary fibrosis. The new genetic model provides new insights into IPF pathophysiology and could be an important resource for the identification of biomarkers and therapeutic targets.

\section{PHARMACOTHERAPY}

\section{Restoring vision in mice}

Mahato, B. et al. Nature https://doi.org/10.1038/ s41586-020-2201-4 (2020)

Retinopathies can result in the death of photoreceptors and irreversible vision loss. Stem-cell therapies to replace lost cells are promising, but they are time-consuming, costly and still far off routine clinical practice.

A new study published in Nature shows that fibroblasts can be directly converted into rod photoreceptor-like cells by using a cocktail of five compounds (valproic acid, CHIR99021, RepSox, forskolin, and IWR1). Transplantation of chemically induced photoreceptor-like cells (CiPCs) into the subretinal space in a mouse model of retinal degeneration partially restored pupil reflex and visual function. CiPCs, which bypass the requirement of pluripotent stem cells, are promising cell-replacement candidates for vision restoration strategies.

\section{PHARMACOTHERAPY}

\section{A multi-species pipeline for drug discovery \\ Volpatti, J.R. et al. eLife 9, pii: e52946 (2020)}

No treatments are available for Ryanodine receptor type I-related myopathies (RYR1-RMs), a group of childhood muscle diseases caused by mutations in the RYR 1 gene and associated with severe disabilities and early mortality.

A study published in eLife describes the development of a multi-system pipeline to identify new therapeutic targets for RYR1-RM. Using this platform, the investigators screened several thousand compounds and tested their efficacy across nematode, zebrafish, and mammalian cell models. The pipeline identified p38 inhibitors as a new class of potential modifiers of RyR1 signaling. This platform could be applied to identify new compounds for RYR1-RM therapy and could be applicable to a broad range of neuromuscular disorders.

https://doi.org/10.1038/s41684-020-0569-2

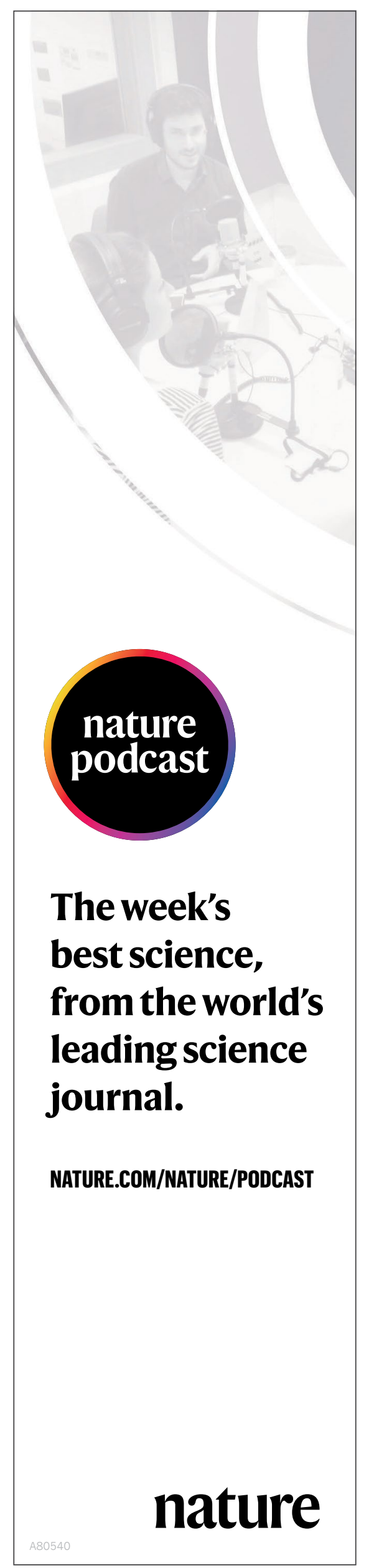

